\title{
EFEITO DO TRATAMENTO DE SUPERFÍCIE DE UMA CERÂMICA ALUMINIZADA INFILTRADA DE VIDRO SOBRE A RESISTÊNCIA À MICROTRAÇÃ̃O
}

\author{
EFFECT OF SURFACE TREATMENT OF A GLASS-INFILTRATED ALUMINA \\ CERAMIC ON THE MICROTENSILE BOND STRENGTH
}

\author{
Silvia Masae de Araujo MICHIDA \\ Estagiária da Disciplina de Prótese Parcial Fixa - UNESP, São José dos Campos/SP. \\ Luiz Felipe VALANDRO \\ Professor Assistente de Prótese Dental do Dep. de Odontologia Restauradora - UFSM/RS. \\ Mestre e Doutorando em Prótese - UNESP, São José dos Campos/SP.

\section{Silvia YOSHIGA} \\ Estagiária da Disciplina de Prótese Parcial Fixa - Universidade de Okayama, Japão.

\section{Osvaldo Daniel ANDREATTA FILHO} \\ Mestre e Doutorando em Prótese - UNESP, São José dos Campos/SP. \\ Ivan BALDUCCI \\ Professor Assist. do Dep. de Odontologia Social e Clínica Infantil - UNESP, São José dos Campos/SP. \\ Marco Antonio BOTTINO \\ Professor Adj. do Dep. de Materiais Odontológicos e Prótese - UNESP, São José dos Campos/SP.
}

\begin{abstract}
E ste estudo avaliou a resistência à microtração entre um cimento resinoso e uma cerâmica submetida a três tratamentos de superfície. Foram confeccionados 12 blocos da cerâmica In-Ceram Alumina (VITA) e 12 blocos de resina composta (Clearfil APX, Kuraray) com dimensões de $6 \mathrm{~mm}$ x $6 \mathrm{~mm}$ x $5 \mathrm{~mm}$. A superfície da cerâmica foi polida com lixas d'água $n^{\circ} 600,800$ e 1200 sob refrigeração, e os blocos foram divididos em três grupos conforme o tratamento superficial: Grupo 1 - Jateamento com óxido de alumínio $110 \mu \mathrm{m}$; Grupo 2 - Sistema Rocatec (3M/ ESPE): jateamento com óxido de alumínio $110 \mu \mathrm{m}$ (Rocatec-pre powder) e com sílica (Rocatec-plus powder) + silano (Rocatec-Sil); Grupo 3 - Sistema CoJet (3M/ESPE): jateamento com partículas de sílica 30 $\mu \mathrm{m}+$ ESPE-Sil. Os blocos cerâmicos foram cimentados aos de resina composta com o cimento resinoso Panavia F (Kuraray Co) conforme as instruções do fabricante, sob carga de $750 \mathrm{~g}$ por 10min. As amostras foram armazenadas (água destilada/ $37^{\circ} \mathrm{C} / 7$ dias) e seccionadas em dois eixos, $\mathrm{x}$ e y, com disco diamantado sob refrigeração em uma máquina de corte a fim de obter corpos-de-prova $(\mathrm{CP})$ com $0,6 \pm 0,1 \mathrm{~mm}^{2}$ de área adesiva $(\mathrm{n}=36)$. Os CP foram fixados em dispositivo adaptado para o teste de microtração e tracionados (velocidade: $0,5 \mathrm{~mm} \cdot \mathrm{min}^{-1}$ ) na máquina de ensaio universal EMIC. Os resultados $(\mathrm{MPa})$ foram submetidos à análise de variância e ao Teste de Tukey $(\mathrm{p}<0,05)$ [grupo 1 15,36; grupo 2 - 30,98 e grupo 3 - 31,25]. Os grupos 2 (Rocatec) e 3 (CoJet) apresentaram maior resistência adesiva que o grupo 1. Não houve diferença estatística significativa entre os grupos 2 e 3 .
\end{abstract}

UNITERMOS: Resistência à microtração; Cerâmica aluminizada; Tratamento de superfície. 


\section{INTRODUÇÃO}

A Odontologia Restauradora tem sido modificada em diversos aspectos que parecem instituir novas regras às modalidades terapêuticas indiretas. Desde que McLean, Hughes $^{12}$, em 1965, introduziram uma nova proposta de sistema cerâmico, diferente dos sistemas até então empregados, a reabilitação protética adotou um rumo promissor no sentido das restaurações estéticas "metal-free".

Embora as características mecânicas tenham tido e merecido pesquisas vastas, a fim de possibilitar melhorias em situações clínicas abrangendo suas indicações, uma preocupação evidente diz respeito à compatibilidade de união de restaurações cerâmicas a estrutura dental.

Inúmeros estudos na área de adesão dental 4,13,14 permitiram avanços consideráveis no processo adesivo de materiais aos tecidos duros dentais, especialmente com o advento de monômeros hidrófilos, os quais têm capacitado diversos procedimentos clínicos antes realizados de forma limitada.

Atualmente o processo de união das cerâmicas feldspáticas aos cimentos resinosos também parece estar bem estabelecido, visto que a união é proporcionada pelo condicionamento com ácido fluorídrico, potencializada pelo agente silano. Ambos têm a propriedade de aumentar a molhabilidade do cimento na superfície, facilitando o contato com os cimentos resinosos. Além disso, o silano desempenha função de ligação entre a sílica contida na cerâmica e a matriz orgânica dos cimentos resinosos, através de uniões siloxanas. $5,10,18$

Entretanto, nem o condicionamento com ácido fluorídrico ou sulfúrico, nem a silanização, têm tido eficácia em proporcionar união entre cerâmicas aluminizadas com baixo teor de sílica aos cimentos resinosos. Estudos têm comprovado esta ineficiência, pela incapacidade de degradar a microestrutura compacta da cerâmica com alto conteúdo de alumina, como ocorre com a dissolução da matriz vítrea das porcelanas feldspáticas. ${ }^{3,4}$

Desta forma, parece importante investigar alguns métodos de tratamento de superfície da cerâmica In-Ceram Alumina, haja vista a ausência de um procedimento eficiente para a mesma. Mesmo sabendo que alguns trabalhos científicos já tenham sido feitos nesse sentido, mostrando indicadores positivos para alguns métodos de tratamento, entendemos, criticamente, que a metodologia de ensaio empregada nestes trabalhos tem sido muito discutida, colocando em dúvida seus resultados. ${ }^{16,19,20,21 .}$

A proposta deste estudo foi analisar o efeito de tratamentos da superfície cerâmica sobre a resistência a microtração entre uma cerâmica aluminizada e um cimento resinoso.

\section{MATERIAL E MÉTODO}

O teste de microtração foi utilizado para avaliar três (3) métodos de tratamento de superfície sobre a cerâmica aluminizada In-Ceram ${ }^{\circledR}$ Alumina (VITA Zahnfabrik,
Germany), quando cimentada com o cimento resinoso Panavia F (Kuraray Co., Osaka - Japan) a uma superfície de resina composta.

Inicialmente, um bloco retangular de resina acrílica com dimensões de $6 \mathrm{~mm}$ de espessura, $6 \mathrm{~mm}$ de altura e $15 \mathrm{~mm}$ de comprimento foi usinado em torno. Seguindo as instruções do fabricante, quatro blocos cerâmicos foram confeccionados considerando as dimensões do bloco de acrílico.

Todos os blocos cerâmicos foram analisados superficialmente em estereoscópio - 50x (Modelo Stemi 2000C, Carl Zeiss). Executou-se verificação radiográfica a fim de observar possíveis falhas internas (bolhas). A presença de defeitos determinou sua exclusão do estudo. A superfície cerâmica, escolhida para futura cimentação, foi polida em Politriz - lixas d'água de granulações $n^{\circ} 600,800$ 1200 (Della Bona, Anusavice, Shen ${ }^{5}$, 2000).

Em seguida, cada bloco cerâmico foi dividido em três porções ( $6 \times 6 \times 5 \mathrm{~mm}$ ), perfazendo um total de 12 blocos menores, os quais foram distribuídos aleatoriamente nos grupos experimentais - 4 blocos por grupo.

A fim de duplicar os blocos cerâmicos, esses foram moldados com silicona de adição (Express, 3M/ESPE USA). Nos moldes obtidos, uma resina composta (Clearfil APX, Kuraray CO. - Japão) foi condensada em camadas (2 $\mathrm{mm}$ ) e fotopolimerizada por 40 segundos (XL 3000 - 3M/ ESPE - USA), até o preenchimento, obtendo-se um bloco de polímero $(6 \mathrm{~mm} \times 6 \mathrm{~mm} \times 5 \mathrm{~mm})$ para cada bloco cerâmico. $^{7,8}$

Após, as superfícies dos blocos cerâmicos foram assim tratadas:

- TRATAMENTO 1 (controle) - microjateamento (Micro-Etcher - Danville Inc., Danville - USA) com partículas de óxido de alumínio (granulação de $110 \mu \mathrm{m}$ ), com ângulo de incidência perpendicular e a uma distância padrão de $10 \mu \mathrm{m}$ da superfície, durante 20 segundos com pressão de 2,8 bars sendo, posteriormente, lavadas em ultra-som por 3 minutos em solução salina ( $\mathrm{NaCl} 0,9 \%)$.

- TRATAMENTO 2 (ROC) - Sistema Rocatec, 3M/ ESPE - as cerâmicas foram jateadas com partículas de óxido de alumínio $110 \mu \mathrm{m}$, a uma distância padrão de $10 \mu \mathrm{m}$ e perpendicular à superfície, durante 20 segundos com pressão de 2,8 bars (Rocatec-pre powder). Seguiu-se o microjateamento com partículas especiais de sílica de $110 \mathrm{~mm}$ (Rocatec-plus powder). Por fim, aplicou-se o silano (Rocatec-Sil) ${ }^{6}$.

- TRATAMENTO 3 (CoJet) - Sistema CoJet, 3M/ESPE - inicialmente, a superfície foi tratada como o condicionamento 1. Em seguida, esta foi submetida ao jateamento com partículas de sílica $(30 \mu \mathrm{m})$, seguindo as mesmas normas operatórias dos condicionamentos 1 e 2; seguiu-se a aplicação do agente silano ESPE-Sil.

Cada bloco cerâmico foi unido ao bloco correspondente de resina composta (Figura 1), empregando o cimento resinoso Panavia F (Kuraray, CO), que foi manipulado, conforme as recomendações do fabricante, e aplicado sobre a superfície tratada do bloco cerâmico. O conjunto cerâmicacimento-polímero foi posicionado em prensa com a interface 
adesiva mais perpendicular possível de carga vertical (750g), por 10min.

Seguiu-se a remoção dos excessos, fotopolimerização por 40 segundos (unidade foto-ativadora XL 3000 - 3M/ ESPE) e aplicação do Oxyguard em todas as margens das interfaces. Passado o tempo, os blocos foram lavados com jato de ar-água e armazenados em solução salina $(\mathrm{NaCl}$ $0,9 \%$ ) a $37^{\circ} \mathrm{C}$ durante $7 \operatorname{dias}^{7,8}$.

Os blocos cimentados foram, então, fixados com cianoacrilato (Super-Bonder Gel - Loctite) em uma base metálica, que por sua vez foi acoplada em uma máquina de corte (Andreatta Filho, Nishioka, Almeida ${ }^{2}$, 2000). Inicialmente, foi realizada uma secção de aproximadamente $0,5 \mathrm{~mm}$ que foi descartada, pois o excesso ou a ausência de cimento na interface influenciaria os resultados (Figuras 2 e 3). Em seguida, 3 secções de $0,8 \pm 0,1 \mathrm{~mm}$ foram realizadas. Cada secção obtida foi girada em $90^{\circ}$ e fixada na base metálica, para que fossem realizada 3 secções de $0,8 \pm 0,1 \mathrm{~mm}$, perfazendo um total de 9 corpos-de-prova (CP) por bloco cimentado. Portanto, cada grupo foi constituído por $36 \mathrm{CP}$, que apresentaram as seguintes características (Figura 3): a - forma retangular; b - Interface quadrangular (simétrica) com área aproximadamente de $0,6 \mathrm{~mm}^{2}, \mathrm{c}$ comprimento de $12 \pm 0,1 \mathrm{~mm}$. ${ }^{5,15,17}$

Cada CP foi fixado com adesivo cianoacrilato nas hastes

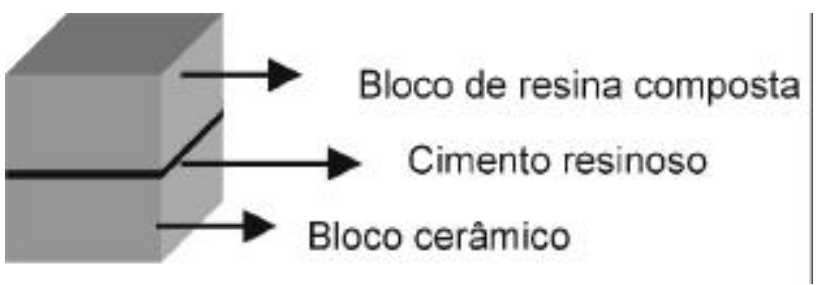

FIGURA 1- Blocos de resina composta e cerâmica cimentados
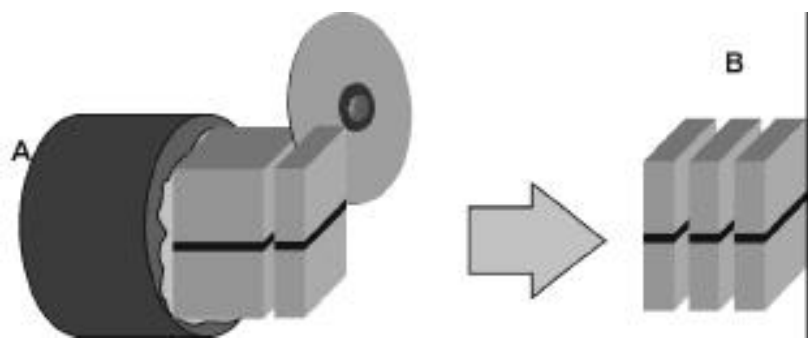

FIGURA 2- Secção do bloco (A); três fatias obtidas (B)
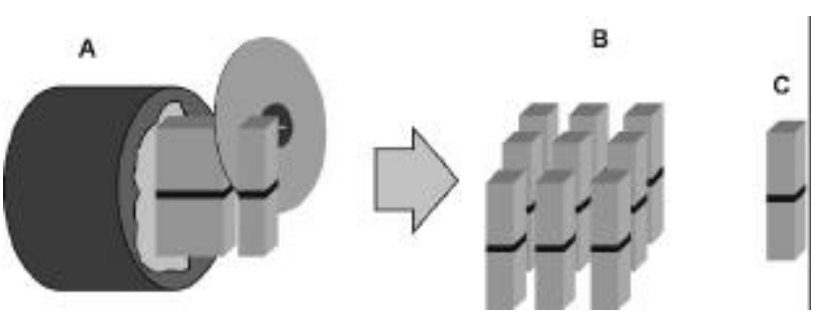

FIGURA 3- Secção das fatias (A); CP obtidos a partir de 3 fatias (B); formato do $\mathrm{CP}(\mathrm{C})$ de um paquímetro adaptado (Vernier Calipers, Starrett) para o ensaio, de modo que a área adesiva se posicionasse o mais perpendicularmente possível à força aplicada, a fim de evitar forças de dobramento na interface. Somente as extremidades do CP foram fixadas, de forma que a zona adesiva permanecesse localizada entre as duas garras do dispositivo (Figura 4). Este conjunto (paquímetro-CP) foi fixado na máquina de ensaios universal (Processo Fapesp n 2001/ 13978-1) e submetido à tração (velocidade de 0,5 mm.min 1) até o rompimento do CP. ${ }^{5,17}$

As amostras fraturadas foram analisadas sob estereomicroscópio com o propósito de verificar o modo de falha (adesiva, coesiva ou mista).

Os dados obtidos (MPa) foram tabulados e analisados estatisticamente, pela análise de variância (ANOVA) e pelo teste de Tukey (nível de significância de 5\%).

\section{RESULTADOS}

Os valores médios das resistências de união e desvio padrão estão expressos na figura 5, para os três métodos de tratamento.

A Análise de Variância indicou que as médias diferiram entre si (Tabela 1 e Figura 5). De acordo com o teste de Tukey, o grupo $2-$ ROC (Média $=30,98 \mathrm{MPa}$ ) e o grupo 3 - CoJet (Média = 31,25 MPa) foram estatisticamente semelhantes e maiores que o grupo 1 - JAT (Média = 15,36 $\mathrm{MPa}$ ).

Conforme a análise estatística, foi possível observar o significante aumento na resistência à microtração quando a cerâmica foi submetida aos condicionamentos que permitem a silicatização da superfície de união (grupos 2 e 3 ).

Após análise dos modos de falha, observou-se que todas as amostras (100\%), independentemente dos grupos investigados, apresentaram fratura do tipo adesiva na interface cimento resinoso / cerâmica.

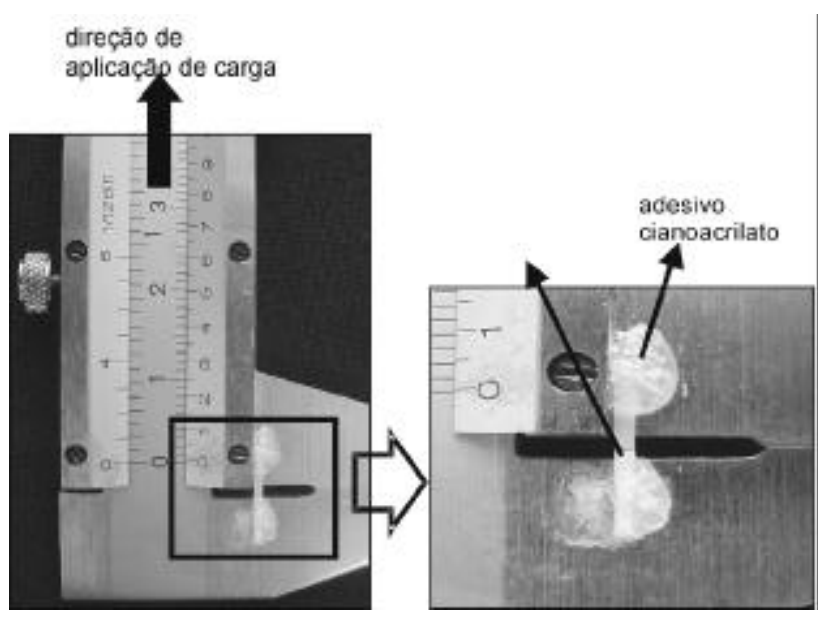

FIGURA 4- Corpo-de-prova fixado nas hastes do paquímetro adaptado 


\section{DISCUSSÃO}

O presente estudo observou que para se obter maior resistência de união entre a cerâmica aluminizada infiltrada de vidro In-Ceram Alumina e o cimento resinoso Panavia F é importante tratar a superfície cerâmica com métodos que proporcionem a deposição de sílica na superfície, como os sistemas de tratamento Rocatec [grupo 2] e CoJet [grupo 3] (Figura 5).

Outros estudos foram conduzidos a fim de avaliar a resistência de união entre agentes cimentantes e cerâmicas aluminizadas, embora tenham empregado metodologias diferentes, especialmente no que se refere aos métodos de ensaio (tração convencional e cisalhamento). Todos observaram que estes métodos de silicatização (Sistema Rocatec) permitiram aumento significativo na resistência de união, comparado aos métodos tradicionais de jateamento com partículas de $\mathrm{Al}_{2} \mathrm{O}_{3}$ ou ácido fluorídrico 3,7,8,11.

$\mathrm{O}$ silano desempenha função de ligação entre a sílica contida na cerâmica e a matriz orgânica dos cimentos resinosos (uniões siloxanas), além de aumentar a molhabilidade dos agentes de união na superfície e assim otimizar a interação microscópica entre o substrato e esses agentes $^{5,10,18}$. Esta relação presença de sílica/silano e capacidade de união está bem estabelecida em porcelanas feldspáticas.

A cerâmica In-Ceram Alumina apresenta 80 a $82 \%$ de alumina e menos de $5 \%$ de sílica na sua composiçãoo. Estas características micro-estruturais limitam uma união confiável a cimentos resinosos quando tratada pelos métodos tradicionais, tais como com ácido fluorídrico ou jateamento com partículas de $\mathrm{Al}_{2} \mathrm{O}_{3}$.

O sistema Rocatec de deposição de sílica é executado em três fases: $1^{\circ}$ - jateamento com partículas de $\mathrm{Al}_{2} \mathrm{O}_{3}$ (Rocatec-Pre powder); $2^{\circ}$ - jateamento com partículas especiais de sílica (Rocatec-Plus powder); $3^{\circ}$ - silanização com Rocatec-Sil. O estudo de Kern, Thompson ${ }^{9}$, em 1993, observou um aumento significativo de sílica na superfície da cerâmica In-Ceram $(15,8 \%$ - 19,7\%) depois do jateamento com Rocatec-Plus, comparado às amostras da mesma cerâmica jateadas apenas com partículas de $\mathrm{Al}_{2} \mathrm{O}_{3}$. Esse estudo sugeriu que a superfície silicatizada pelo sistema Rocatec poderia desenvolver melhor resistência de união entre a cerâmica In-Ceram e cimentos resinosos, tendo em vista o aumento do conteúdo de sílica e a possibilidade de

TABELA 1- Média e desvios padrões dos valores de resistência adesiva

\begin{tabular}{lllll}
\hline Grupos & Média \pm DP & \multicolumn{2}{c}{ Conjunto } \\
\hline 1: $\mathrm{Al}_{2} \mathrm{O}_{3}$ & $15,36 \pm 5,26$ & $\mathrm{~A}$ & - \\
2: $\mathrm{ROC}$ & $30,98 \pm 5,42$ & - & $\mathrm{B}$ \\
3: Cojet & $31,25 \pm 9,12$ & - & $\mathrm{B}$ \\
\hline
\end{tabular}

* Médias seguidas de letras iguais não diferem estatisticamente. interação com o agente silano (Rocatec-Sil) e este com materiais resinosos. Este aumento na capacidade de união tem sido comprovado em diversos estudos ${ }^{7,8,11}$.

Embora o presente estudo tenha avaliado a capacidade de união de uma cerâmica aluminizada infiltrada de vidro (In-Ceram Alumina) a um cimento resinoso (Panavia F), é possível considerar que todos estes argumentos no sentido de estabelecer os métodos de silicatização como procedimento indispensável para a referida união, poderiam ser estendidos para outras cerâmicas ácido-resistentes e outros cimentos resinosos ${ }^{7,9,11}$.

A cerâmica ProCera AllCeram apresenta a mesma dificuldade de tratamento de superfície que a cerâmica InCeram, uma vez que apresenta elevado conteúdo de alumina (In-Ceram Alumina: $\pm 80 \%$; ProCera AllCeram: $\pm 99,9 \%$ ) e baixo conteúdo de sílica. ${ }^{1}$ Neste sentido, alguns estudos observaram que a deposição de sílica por meio do sistema Rocatec na cerâmica ProCera AllCeram também promove um aumento significativo na resistência de união a cimentos resinosos, embora estes estudos tenham usado metodologias de ensaio diferentes da utilizada por nós ${ }^{7,8}$.

O sistema CoJet de deposição de sílica é um método de tratamento similar ao sistema Rocatec: $1^{\circ}$ - jateamento com $\mathrm{Al}_{2} \mathrm{O}_{3} ; 2^{\circ}$ - jateamento com óxido de sílica; $3^{\circ}$ - silanização com ESPE-Sil.

O presente estudo observou aumento significante da resistência de união da cerâmica tratada pelo sistema CoJet (grupo 3), em relação à cerâmica tratada convencionalmente (grupo 1). Os mecanismos de promoção da união por este sistema estão baseados nos mesmos argumentos relatados ao sistema Rocatec.

No que se refere à resistência de união de outros cimentos resinosos a base de Bis-GMA, estudos têm demonstrado alta resistência de união entre cerâmicas aluminizadas e a base de zircônia silicatizadas pelo sistema Rocatec e estes cimentos $3,7,8,11$.

Entretanto, de acordo com as considerações de Van Noort, et al..$^{20}(1989)$, Van Noort, et al. ${ }^{19}(1991)$, Sano, et al. ${ }^{15}$ (1994), Versluis, et al. ${ }^{21}$ (1997) e Schreiner, et al. ${ }^{16}$ (1998), as metodologias que avaliaram a resistência de união entre cerâmicas ácido-resistentes e cimentos resinosos não são recomendadas.

O presente estudo utilizou uma metodologia considerada confiável, conforme estudos conduzidos a fim de comparar

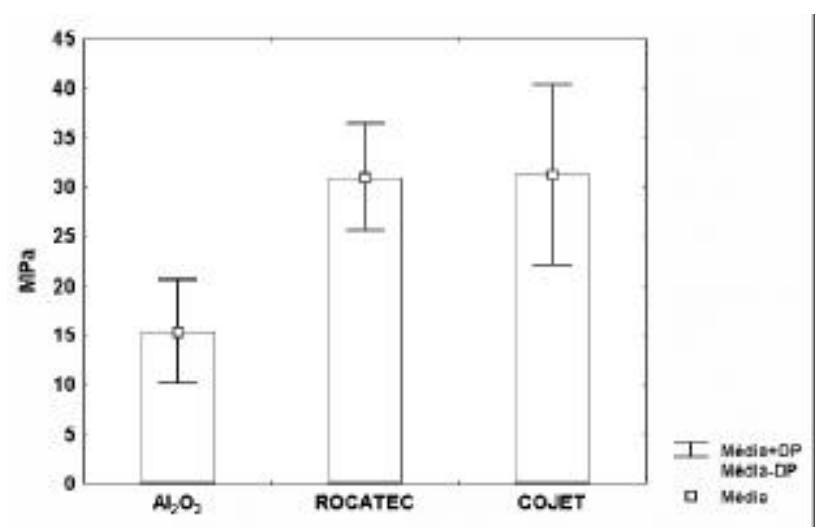

FIGURA 5- Média e desvio padrão dos dados obtidos 
diferentes métodos para investigar a resistência de união entre diferentes materiais ${ }^{15,16,19,20,21}$. Portanto, é oportuno discutir alguns aspectos experimentais que julgamos cientificamente importantes:

1) A cimentação dos blocos cerâmicos aos de resina composta e não à dentina é justificada pelas variações microestruturais deste substrato (procedência, armazenagem, idade), que poderiam gerar variabilidade dos resultados, ao passo que os blocos de resina composta foram obtidos sob idênticas condições experimentais, e assim sem variações.

2) O emprego de maior número de dentes e $\mathrm{CP}$ por grupo, a fim de diminuir o desvio-padrão, é importante quando o ensaio de microtração é utilizado para estudar a resistência de união de sistemas adesivos ao substrato dentinário, devido sua variabilidade. O presente trabalho avaliou a resistência a microtração de amostras cerâmicas obtidas sob idênticas condições de processamento (tempo de sinterização da alumina, ciclo de infiltração do vidro), permitindo afirmar que todos os blocos cerâmicos apresentaram a mesma microestrutura, não influenciando os resultados.

\section{CONCLUSÃO}

A resistência de união dos grupos em que a cerâmica foi silicatizada pelos sistemas Rocatec ou CoJet foi maior que a resistência de união da cerâmica tratada somente por meio do jateamento com óxido de alumínio.

\section{ABSTRACT}

This study evaluated the microtensile bond strength of a resin composite to a ceramic submitted to three surface treatments. Twelve glass-infiltrated alumina ceramic blocks (In-Ceram Alumina, VITA) and twelve resin composite blocks (Clearfil APX, Kuraray) with dimensions of $6 \mathrm{~mm} x$ $6 \mathrm{~mm} \times 5 \mathrm{~mm}$ were made. The surface of the ceramic was wet-grounded with \# 600, 800 and 1200-grid sandpaper, and the blocks were divided in three groups: Group 1 sandblasting with aluminum oxide - particles $110 \mu \mathrm{m}$ (Micro-Etcher, Danville); Group 2 - Rocatec System (ESPE): Tribochemical silica coating (Rocatec pre-powder + Rocatec-Plus powder + Rocatec-Sil); Group 3 - CoJet System (3M/ESPE): silica coating (silica oxide + ESPE$\mathrm{Sil}$ ). The ceramic blocks were cemented to the resin composite blocks with Panavia F (Kuraray Co), following the manufacturer's instructions, under load of $750 \mathrm{~g}$ for $10 \mathrm{~min}$. The samples were stored (distilled water / 7 days / $37^{\circ} \mathrm{C}$ ) and sectioned in two axis, $\mathrm{x}$ and $\mathrm{y}$, with diamond disk under cooling in order to obtain samples $(\mathrm{S})$ with $0.6 \pm$ $0.1 \mathrm{~mm}^{2}$ of adhesive area $(n=36)$. The $S$ were attached in adapted device for the microtensile test that was performed at an universal testing machine (EMIC), at a speed of $1 \mathrm{~mm} /$ min. The results (MPa) were submitted to ANOVA and Tukey's test ( $\mathrm{p}<0.05)$ : $\mathrm{G} 1-15.36 ; \mathrm{G} 2-30.98 ; \mathrm{G} 3-31.25$. Groups 2 (Rocatec) and 3 (CoJet) presented larger bond strength than group 1 . There was no statistical difference between group 2 and group 3.

UNITERMS: Microtensile bond strength; Aluminabased ceramic; Surface treatment.

\section{AGRADECIMENTOS}

Esta pesquisa foi apoiada pela Fundação de Amparo à Pesquisa do Estado de São Paulo (FAPESP) - Processo $n^{\circ}$ 02/01536-7. Bolsa de Iniciação Científica.

\section{REFERÊNCIAS BIBLIOGRÁFICAS}

1- Andersson M, Odén A. A new all-ceramic crown - a densesintered, high-purity alumina coping with porcelain. Acta Odontol Scand 1993; 51: 59-64.

2- Andreatta Filho OD, Nishioka RS, Almeida EES, Construção de um torno mecânico para realizar reparos dentais padronizados. In: Anais da $17^{\text {a }}$ Reunião Anual da Sociedade Brasileira de Pesquisa Odontológica - SBPqO: 2000; set. 02 - 06; Águas de Lindóia (SP); p. 17. (Resumo ${ }^{\circ}$ 1047)

3- Awliya W, Odén A, Yaman P, Dennison JB, Razzoog ME. Shear bond strength of resin cement to densely sintered high-purity alumina with various surface conditions. Acta Odontol Scand 1998; 56: $9-13$.

4- Buonocore MG. A simple method of increasing the adhesion of acrylic filling materials to enamel surfaces. J Dent Res 1955; 34: $849-53$

5- Della Bona A, Anusavice KJ, Shen C. Microtensile strength of composite bonded to Hot-pressed Ceramics. J Adhesive Dent 2000; 2:305-13.

6- Guggenberger R. Das Rocatec-System - haftung durch tribochemischi beschichtung. Dtsch Zahnärztl Z 1989; 44:874-6.

7- Kern M, Thompson VP. Bonding to glass infiltrated alumina ceramic: adhesive methods and their durability. J Prosthet Dent 1995; 73:240-9.

8- Kern M, Thompson VP. Sandblasting and silica coating of a glass-infiltrated alumina ceramic: volume loss, morphology, and changes in the surface composition. J Prosthet Dent 1994; 71:45361 .

9- Kern M, Thompson VP. Tensile bond strength of new adhesive systems to In-Ceram ceramic [Abstract $n^{\circ}$ 2124]. J Dent Res, 1993; $72: 369$.

10- Lu R, Harcourt JK, Tyas MJ, Alexander B. An investigation of composite resin/porcelain interface. Aust Dent J 1992; 37:129.

11- Madani M, Chu FCS, Mcdonald AV, Smales RJ. Effects of surface treatments on shear bond strengths between a resin cement and an alumina core. J Prosthet Dent 2000; 83: 644-7. 
12- Mclean JW, Hughes TH. The reinforcement of dental porcelain with ceramic oxides. Br Dent J 1965; 119:251-67.

13- Nakabayashi N, Pashley DH. Hybridization of dental hard tissues. Tokyo: Quintessence; 2000.

14- Nakabayashi N. Adhesive bonding with 4-meta. In: Internacional Symposium on Adhesives in Dentistry: 1991 jul. 11 - 13; Omaha: 1992. p.125-30.

15- Sano H, Shono Y, Sonoda H, Takatsu T, Ciucchi B, Carvalho $\mathrm{R}$, et al. Relationship between surface area for adhesion and tensile bond strength - evaluation of a micro-tensile bond test. Dent Mater 1994; 10:236-40.

16- Schreiner RF, Chappell RP, Glaros AG, Eick JD. Microtensile testing of dentin adhesives. Dent Mater 1998; 14:194-201.

17- Shono Y, Ogawa T, Terashita M, Carvalho RM, Pashley EL, Pashley DH. Regional measurement of resin-dentin bonding as an array. J Dent Res 1999; 78(2):699-705.

18- Simonsen RJ, Calamia JR. Tensile bond strength of etched porcelain [Abstract $n^{\circ}$ 1154]. J Dent Res 1983; 62:297.

19- Van Noort R, Cardew G, Howard IC, Noroozi S. The effect of local interfacial geometry on the measurement of the tensile bond strength to dentin. J Dent Res 1991; 70: 889-93.

20- Van Noort R, Noroozi S, Howard IC, Cardew G. A critique of bond strength measurements. J Dent 1989; 12(2):61-7.

21. Versluis A, Tantbirojn D, Douglas WH. Why do shear bond tests pull out dentin? J Dent Res, 1997; 76:1298-307.

Recbido para publicação em: 30/01/2003

Enviado para reformulações em: 19/03/2003

Pronto para publicação em: 17/07/2003

Endereço do autor correspondente Prof. Assist. LUIZ FELIPE VALANDRO:

Av. Francisco José Longo, 777

Bairro São Dimas - Cep.: 12245-000

São José dos Campos - SP

E-mail: lfvalandro@smail.ufsm.br

Fone: (12) 3947-9056

Fax: (12) 3947-9000 